\title{
Abordaje de hernias poco frecuentes por cirugía de mínimo acceso: serie de casos
}

\author{
Minimally invasive surgery as an approach for less \\ frequent type of hernias: case series
}

\author{
Ana Paula Ruiz-Funes Molina, ${ }^{*}$ Jorge Farell Rivas, ${ }^{\star}$ Antonio Marmolejo Chavira, ${ }^{\star}$ \\ Andrés de Jesús Sosa López, ${ }^{*}$ Alejandro Cruz Zárate* \\ * Hospital Central Sur de Alta Especialidad PEMEX. México.
}

\section{RESUMEN}

Introducción: La mayor parte de las hernias abdominales se localizan en la línea media; sin embargo, existen hernias en sitios menos comunes o con componentes atípicos. Objetivo: Presentar una serie de casos con hernias raras y comparar nuestros resultados con los reportados en la literatura para reafirmar la seguridad y efectividad del abordaje laparoscópico en la reparación de este tipo de hernias. Material y métodos: Se realizó abordaje laparoscópico en siete pacientes con diagnóstico de hernia poco frecuente en un hospital de la Ciudad de México. Se incluyeron pacientes con hernias en distintas localizaciones (lumbar, Spiegel, obturatriz, inguinal) clasificadas como raras por la localización atípica o por el contenido del saco herniario. Resultados: Se realizó cirugía laparoscópica a siete pacientes con diagnóstico de hernia rara. Se incluyó un paciente con hernia de Petit, al cual se le realizó una plastia retromuscular lateral con abordaje e-TEP, tres pacientes con hernia de Spiegel, dos por abordaje TAPP y uno e-TEP con TAR ipsilateral al defecto, y uno con hernia obturatriz, a quien se le realizó una plastia TAPP. Se operaron dos pacientes de urgencia, uno con hernia de Amyand, a quien se le hizo apendicectomía, y uno con hernia de Richter, al cual se le realizó resección intestinal y anastomosis; en ambos se realizó plastia TAPP. Todos cursaron con adecuada evolución posquirúrgica. Conclusiones: La cirugía laparoscópica ha demostrado ser segura para la reparación de hernias poco frecuentes. Encontramos un tiempo de recuperación y reincorporación a las actividades de la vida cotidiana más cortas; para poder generalizar estos resultados, se requiere de una serie de pacientes mayor.

Palabras clave: Hernia de Spiegel, hernia lumbar, hernia obturatriz, hernia de Amyand, hernia de Richter, hernia atípica, laparoscopía, mínima invasión.

\section{ABSTRACT}

Introduction: Most abdominal wall hernias have a typical clinical onset and are located over the midline, but there's a small group of hernias located in less frequent sites or considered rare because of its components. Objective: The objective of this study is to present a case series of patients with rare hernias repaired by laparoscopy, and compare our results to the reported results by the literature in order to confirm the security and effectiveness of this approach. Material and methods: Laparoscopic surgery was performed in seven patients with rare hernia's diagnosis in a hospital in Mexico City. We included patients with hernias in different locations (lumbar, Spiegel, obturator or inguinal), classified as rare hernias for its location or hernia's sac contents. Results: Minimally invasive surgery was performed in seven patients, six female and one male, all of them with rare hernias. Three patients with Spiegel hernia underwent surgery, two with a TAPP approach and one with an e-TEP with TAR approach. One patient with Petit's hernia was included; lateral e-TEP approach was performed, with retromuscular mesh placement. One patient underwent TAPP approach in order to repair an obturator hernia. Two patients needed urgent surgery. One had an Amyand hernia and appendectomy was performed after the TAPP repair, the other had a Richter's hernia and bowel resection with anastomosis was done after the TAPP hernia repair. Every patient had a good postoperative outcome and none of them have shown relapse or long term complications. Conclusions: Laparoscopic surgery is safe for less frequent hernia's repair. It is associated to a faster recovery and shorter hospital stay, as well as less complications' risk.

Keywords: Spiegel hernia, lumbar hernia, obturator hernia, Amyand hernia, Richter hernia, atypical hernia, laparoscopy, minimally invasive. 


\section{INTRODUCCIÓN}

La reparación de hernias de la pared abdominal es actualmente uno de los procedimientos realizados con mayor frecuencia. La mayor parte de los defectos abdominales tienen una presentación clínica típica localizada en la línea media; sin embargo, existen hernias localizadas en sitios menos comunes o con componentes atípicos. ${ }^{1}$ Dentro del grupo de hernias raras o poco frecuentes de localización atípica se encuentran: hernia de Spiegel; obturatriz; y lumbares. Las hernias con componentes poco habituales incluyen a la hernia de Amyand, Garengeot y Richter, entre otras.

Cada una de estas hernias tiene manifestaciones clínicas diferentes y con frecuencia se presentan con síntomas que se traslapan con otras patologías. Por tal motivo, se requiere de una alta sospecha clínica y, en muchas ocasiones, apoyo de estudios de imagen para realizar el diagnóstico y planeación del procedimiento quirúrgico.

La hernia de Spiegel debe su nombre a su localización en el sitio de debilidad de fascia entre el recto abdominal y la línea semilunar, la fascia de Spiegel. ${ }^{2}$ Tiene una frecuencia de aproximadamente 0.1 a $2.5 \%$ y se presenta más comúnmente en mujeres. Este tipo de hernia puede ser primaria o incisional. ${ }^{3,4}$ Los síntomas varían desde la presencia de dolor abdominal y el aumento de volumen en la pared anterolateral del abdomen, hasta la incarceración o la obstrucción intestinal. ${ }^{5}$ En estos pacientes es indispensable la presencia de un estudio de imagen que confirme el diagnóstico, por la variabilidad en la forma de presentación y la baja especificidad de sus síntomas. ${ }^{6,7}$

Las hernias lumbares incluyen a la hernia de Grynfelt, formada en el triángulo lumbar superior, triángulo comprendido entre la doceava costilla, los músculos paraespinales y el músculo oblicuo interno; y la hernia de Petit, formada en el triángulo lumbar inferior, delimitado por la cresta iliaca, el músculo dorsal ancho y el oblicuo externo. ${ }^{8,9}$ Las hernias lumbares tienen una frecuencia menor al $2 \%$ y su forma de presentación es muy variable. Suelen ser asintomáticas, pero pueden producir dolor o aumento de volumen. ${ }^{10}$

Otra hernia de localización atípica, adicional a la hernia de Spiegel y las lumbares, es la hernia obturatriz, que es de seis a nueve veces más frecuente en mujeres que en hombres; esto se debe a que la pelvis es más ancha y el foramen obturatriz tiene un mayor diámetro. Entre los factores asociados al desarrollo de una hernia obturatriz se encuentran: laxitud de los tejidos pélvicos; ausencia de grasa preperitoneal que cubra el agujero obturatriz; e incremento en la presión intraabdominal, factores más comunes en mujeres multíparas. ${ }^{11}$ Este tipo de hernia constituye del 0.07 al $1 \%$ del total de las hernias de pared abdominal. A pesar de su baja frecuencia, tiene una mortalidad entre el 13 y $40 \%$, por lo que su diagnóstico y tratamiento oportunos son muy relevan- tes. ${ }^{12}$ Aunque la sospecha diagnóstica ante la presencia del signo de Howship-Romberg (dolor o parestesia sobre la cara interna del muslo) es alta, en general la presentación clínica es vaga e inespecífica, por lo general no se palpa. Incluso con el apoyo de estudios de imagen, donde la presencia de tumor entre los músculos pectíneo y obturador externo es patognomónico, su diagnóstico resulta complejo. Por lo anterior, con frecuencia debuta como hernia complicada. ${ }^{13}$

Como ya se mencionó antes, también existen hernias poco frecuentes por sus componentes o el contenido de su saco herniario.

Se define como hernia de Amyand a la hernia inguinal cuyo saco herniario contiene al apéndice cecal. Tiene una incidencia de presentación cercana al 1\% y únicamente se asocia con apendicitis aguda en el $0.1 \%$ de los casos. ${ }^{14}$ Cuando el defecto herniario se encuentra en el orificio femoral, se denomina hernia de Garengeot. ${ }^{15,16}$

Cuando el contenido del saco de una hernia incluye el borde antimesentérico del intestino delgado, con o sin compromiso de la vasculatura de una porción de la pared intestinal, se denomina hernia de Richter. Generalmente el defecto es inguinal, aunque se han descrito también en defectos femorales, umbilicales o incisionales del sitio de inserción del puerto laparoscópico..$^{17,18}$

El manejo de elección para todas las hernias mencionadas es la reparación quirúrgica. La selección del abordaje quirúrgico depende del tamaño del defecto, localización, contenido del saco, estado general del paciente, posibilidad de recurrencia, disponibilidad de los recursos y experiencia del equipo quirúrgico. Las hernias lumbares y de Spiegel son por definición hernias complejas; además, generalmente las hernias de contenido atípico se presentan en un escenario de urgencia, considerándose también hernias complejas. ${ }^{19}$

La colocación de material protésico en hernias incarceradas o estranguladas es controversial; sin embargo, en presencia de una cirugía limpia-contaminada, con resección intestinal sin riego de líquido intestinal, no existe contraindicación para su uso. ${ }^{20}$

La cirugía de mínimo acceso ha resultado una herramienta de gran utilidad que muestra un amplio beneficio en el tratamiento de hernias poco frecuentes. Este abordaje permite una localización exacta del defecto anatómico y favorece la realización de una exploración y disección menos traumática de los planos abdominales por medio de incisiones pequeñas, minimizando el dolor posoperatorio.

Entre los abordajes laparoscópicos descritos se encuentran el transabdominal preperitoneal (TAPP) y el totalmente extraperitoneal extendido (e-TEP). Estos abordajes permiten la colocación de una malla retromuscular o preperitoneal, posición favorecida por la fuerza de la presión intraabdominal, la cual empuja la malla contra la aponeurosis anterior, manteniéndola en su posición original. ${ }^{21,22}$ Otra 
ventaja relacionada con la posición de la malla es que no se encuentra en contacto directo con el contenido intraabdominal, por lo que es segura la colocación de una malla de polipropileno; esto reduce el costo del procedimiento.

Derivado de los beneficios antes descritos, la cirugía laparoscópica se asocia con una menor tasa de infección de sitio quirúrgico, disminución del tiempo de estancia intrahospitalaria, recuperación e integración a la vida diaria, lo que impacta positivamente en los costos totales relacionados con el procedimiento. ${ }^{23}$

A continuación, se presenta una serie de casos de hernias poco frecuentes reparadas por abordaje laparoscópico, con el objetivo de comparar resultados con la literatura descrita para reafirmar la seguridad y efectividad del abordaje mínimamente invasivo en este tipo de hernias.

\section{MATERIAL Y MÉTODOS}

Se realizó abordaje laparoscópico en siete pacientes con diagnóstico de hernia poco frecuente en un hospital de la Ciudad de México. Se incluyeron pacientes con diagnóstico de hernia en distintas localizaciones (lumbar, Spiegel, obturatriz, inguinal) clasificadas como raras por la localización atípica o por el contenido del saco herniario.

Se operó un paciente con hernia de Petit (lumbar), dos con hernia de Spiegel incisional, uno con hernia de
Spiegel primaria, uno con hernia obturatriz, uno con hernia de Amyand con defecto inguinal indirecto y un paciente con hernia de Richter con defecto inguinal indirecto. De los siete casos, únicamente los que presentaban hernia de Amyand y hernia de Richter fueron operados de urgencia. En el resto de los pacientes se realizó cirugía electiva.

En dos casos se realizó un abordaje totalmente extraperitoneal extendido (e-TEP). Se realizó liberación del músculo transverso del abdomen (TAR) ipsilateral al defecto en un paciente con diagnóstico de hernia de Spiegel incisional y abordaje retromuscular lateral en uno con diagnóstico de hernia de Petit. En cinco casos se realizó un abordaje transabdominal preperitoneal (TAPP). En los dos casos de urgencia se realizaron procedimientos adicionales: en el caso de la hernia de Amyand se realizó apendicectomía laparoscópica y en el caso de la hernia de Richter se realizó resección intestinal con anastomosis primaria.

\section{RESULTADOS}

Se realizó cirugía laparoscópica en siete pacientes, seis mujeres $(86 \%)$ y un hombre $(14 \%)$, todos ellos con diagnóstico de hernia rara o poco frecuente. La edad promedio de los pacientes fue de 69 años, con un rango de 58 a 84 . Dos procedimientos se realizaron de forma urgente (29\%); el
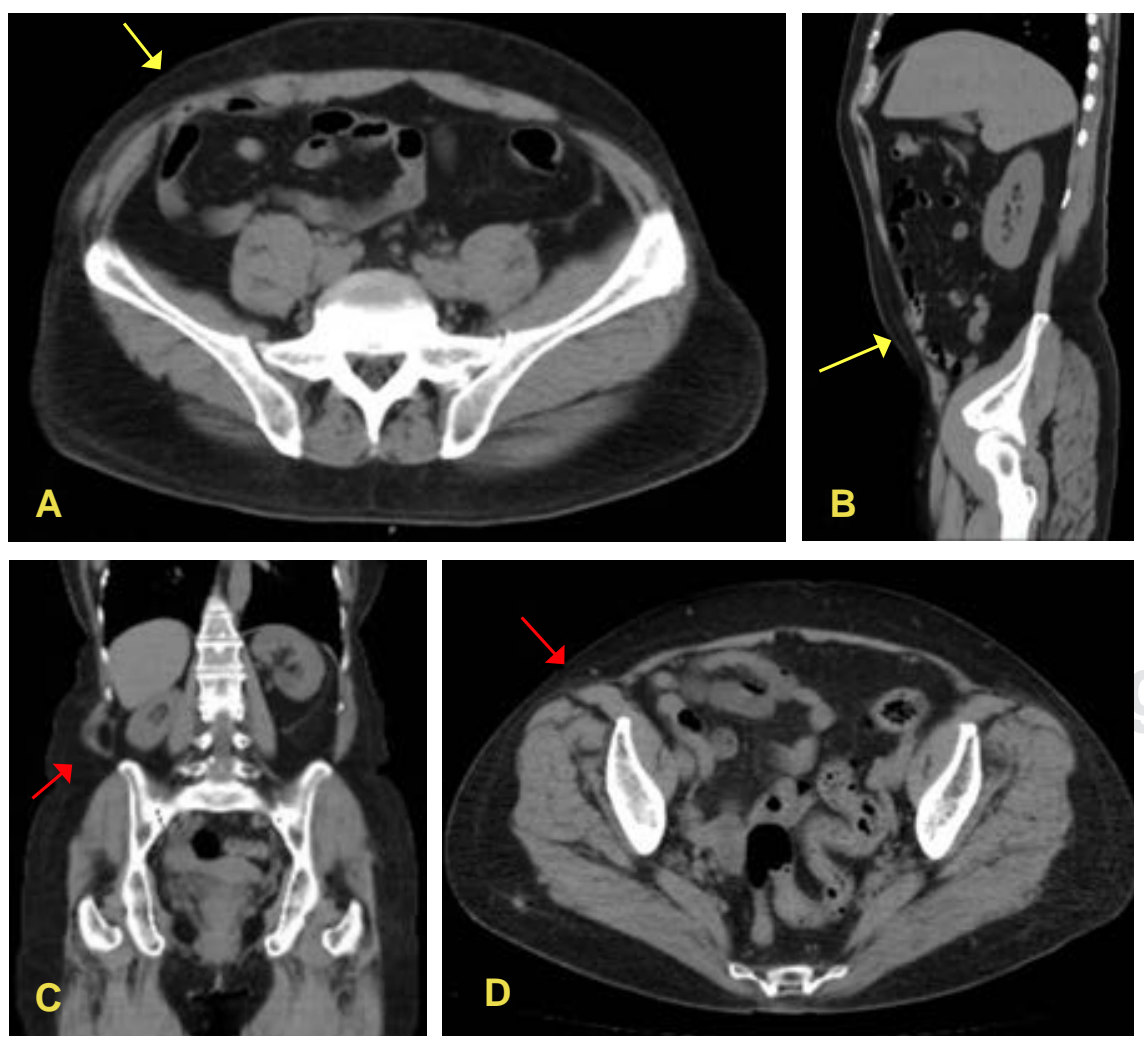

Figura 1:

A) Corte axial de tomografía de paciente 2 con diagnóstico de hernia de Spiegel izquierda (incisional). B) Corte sagital de tomografía de paciente 2 con diagnóstico de hernia de Spiegel izquierda (incisional). C) Corte axial de tomografía de paciente 1 con diagnóstico de hernia de Petit derecha. D) Corte coronal de tomografía de paciente 1 con diagnóstico de hernia de Petit derecha. 

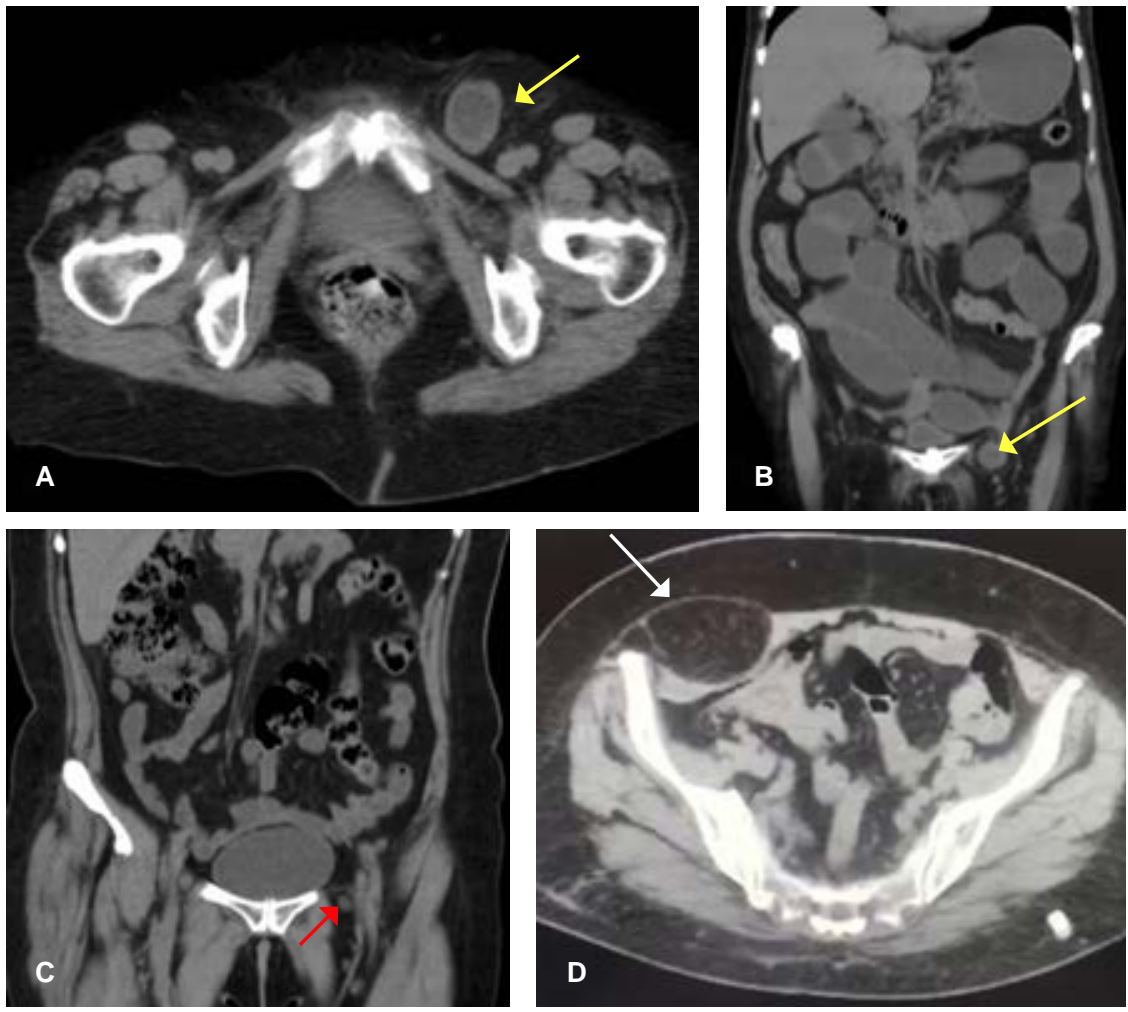

Figura 2:

A) Corte axial de tomografía de paciente 7 con diagnóstico de hernia de Richter (inguinal izquierda). B) Corte coronal de tomografía de paciente 7 con diagnóstico de hernia de Richter (inguinal izquierda). C) Corte coronal de tomografía de paciente 5 con diagnóstico de hernia obturatriz. D) Corte axial de tomografía de paciente 4 con diagnóstico de hernia de Spiegel derecha.

resto fueron procedimientos electivos, con hospitalización un día previo al procedimiento. El 71\% de los casos refirió alguna comorbilidad, entre las que destacaron principalmente diabetes mellitus e hipertensión arterial sistémica. El $57 \%$ de los pacientes incluidos contaba con antecedente de cirugías abdominales previas; sin embargo, únicamente dos de las siete hernias estudiadas (29\%) se clasificaron como hernias incisionales. El paciente con hernia de Amyand contaba con antecedente de plastia inguinal previa, clasificándose como recidivante. El resto de las hernias fueron clasificadas como primarias (Tabla 1).

En todos los casos, se realizó tomografía abdominal simple previa al procedimiento quirúrgico (Figuras 1 y 2). En todos los pacientes se administró una dosis de antibiótico profiláctico (cefalosporina); en ambos pacientes sometidos a cirugía de urgencia, se continuó esquema antibiótico por siete días.

Se operó un paciente con hernia de Petit, localizada del lado derecho. Se realizó una plastia retromuscular lateral, por medio de abordaje e-TEP. Se identificó un defecto de $6 \times 5 \mathrm{~cm}$, mismo que se cerró con sutura de polipropileno del 0 (Tabla 2) y se colocó una malla de polipropileno medianamente pesada de $14 \times 10 \mathrm{~cm}$ en el espacio retromuscular (Tabla 3).

Se identificaron tres pacientes con hernia de Spiegel, dos con lateralidad derecha y una izquierda. En dos de estos casos se realizó una plastia tipo TAPP, se cerró el defecto herniario en ambos y posteriormente se colocó malla de polipropileno de $15 \times 8 \mathrm{~cm}$ y $16 \times 13 \mathrm{~cm}$ en el espacio preperitoneal. Se fijó malla con fijadores absorbibles a la pared abdominal lateral, cuatro sitios de fijación en cada caso, y se realizó cierre de flap peritoneal con poliglactina 910, en ambos casos (Figura 3). En el tercer paciente, donde se identificó una hernia de mayor tamaño $(6 \times 5 \mathrm{~cm})$, se decidió un abordaje totalmente extraperitoneal (e-TEP) y se realizó técnica de liberación del transverso (TAR) ipsilateral

Tabla 1: Características clinicopatológicas de los pacientes con hernias poco frecuentes $(N=7)$.

\begin{tabular}{lc} 
Variables & $\mathrm{n}(\%)$ \\
\hline Femenino & $6(86.0)$ \\
Masculino & $1(14.0)$ \\
Edad en años (promedio) & 69 \\
IMC (promedio) & 26.9 \\
Comorbilidades & $5(71.0)$ \\
Cirugías previas & $4(57.0)$ \\
Hernias primarias & $4(57.0)$ \\
Hernias incisionales & $2(29.0)$ \\
Hernias recidivantes & $1(14.0)$ \\
Cirugías de urgencia & $2(29.0)$ \\
\hline
\end{tabular}


Tabla 2: Localización, tamaño y características del cierre del defecto de hernias poco frecuentes.

\begin{tabular}{|c|c|c|c|c|}
\hline Paciente & Tipo de hernia & Localización del defecto & Tamaño del defecto $(\mathrm{cm})$ & Cierre del defecto \\
\hline 1 & Petit & Lumbar derecha & $6 \times 5$ & Polipropileno 0 \\
\hline 2 & Spiegel & Spiegel izquierda & $1.5 \times 1.5$ & Poliglactina $9102-0$ \\
\hline 3 & Spiegel & Spiegel derecha & $6 \times 5$ & Polipropileno 1 \\
\hline 4 & Spiegel & Spiegel derecha & $4 \times 4$ & Polipropileno 1 \\
\hline 5 & Obturatriz & Obturatriz izquierda & $0.5 \times 1$ & No \\
\hline 6 & Amyand & Inguinal indirecta derecha & $2 \times 1$ & No \\
\hline 7 & Richter & Inguinal indirecta izquierda & $2 \times 2$ & No \\
\hline
\end{tabular}

al defecto. Se realizó cierre del defecto con polipropileno 1 y se colocó una malla de polipropileno medianamente pesada de $21 \times 21 \mathrm{~cm}$ en espacio retromuscular (Figura 4).

En el caso de la hernia obturatriz, por antecedente de dolor inguinal crónico, se decidió abordaje TAPP con la ventaja de realizar laparoscopía diagnóstica. Se identificó un defecto inguinal indirecto izquierdo pequeño de $1 \times$ $1 \mathrm{~cm}$, asociado con una hernia obturatriz con un defecto de $0.5 \times 1 \mathrm{~cm}$. Se redujo el saco en ambos defectos y se colocó una malla de polipropileno de $15 \times 12 \mathrm{~cm}$; se fijó malla al ligamento de Cooper, recto anterior y pared lateral del abdomen, con fijadores absorbibles. Se realizó cierre de flap de peritoneo con sutura barbada (Tablas 2 y 3 ).

En este estudio se incluyeron dos casos sometidos a cirugía de urgencia; en ambos el diagnóstico preoperatorio fue hernia inguinal incarcerada.

En uno de los pacientes se identificó una hernia de Amyand derecha con defecto indirecto cuyo saco contenía al apéndice cecal con diagnóstico de apendicitis no complicada. Se realizó plastia inguinal TAPP con colocación de malla de polipropileno de $12 \times 10 \mathrm{~cm}$, la cual se fijó con fijadores absorbibles a ligamento de Cooper, recto anterior y pared lateral del abdomen. Después se realizó cierre hermético del flap peritoneal con poliglactina 910. Una vez concluida la plastia inguinal, asegurando el cierre adecuado del peritoneo y cobertura completa de la malla, se realizó apendicectomía laparoscópica (Figura 5).

El segundo paciente con indicación para cirugía de urgencia tuvo un diagnóstico de hernia inguinal izquierda incarcerada. Se identificó una hernia recidivante, con un abordaje previo anterior de forma abierta, por lo que se decidió una reparación posterior.

Al realizar la laparoscopía inicial, se encontró una hernia inguinal izquierda recidivante con contenido de borde antimesentérico del íleon (hernia de Richter) y fibrosis por malla previa. Se realizó reducción de saco herniario con evidencia de necrosis del segmento intestinal, sin datos de perforación. Se inició disección de flap peritoneal para continuar con plastia inguinal con técnica transabdominal preperitoneal con colocación de malla. Se colocó malla de polipropileno medianamente pesada de $15 \times 12 \mathrm{~cm}$. Se colocó malla con fijadores absorbibles a ligamento de Cooper, recto abdominal y pared lateral del abdomen. Se cerró peritoneo de forma hermética con surgete continuo
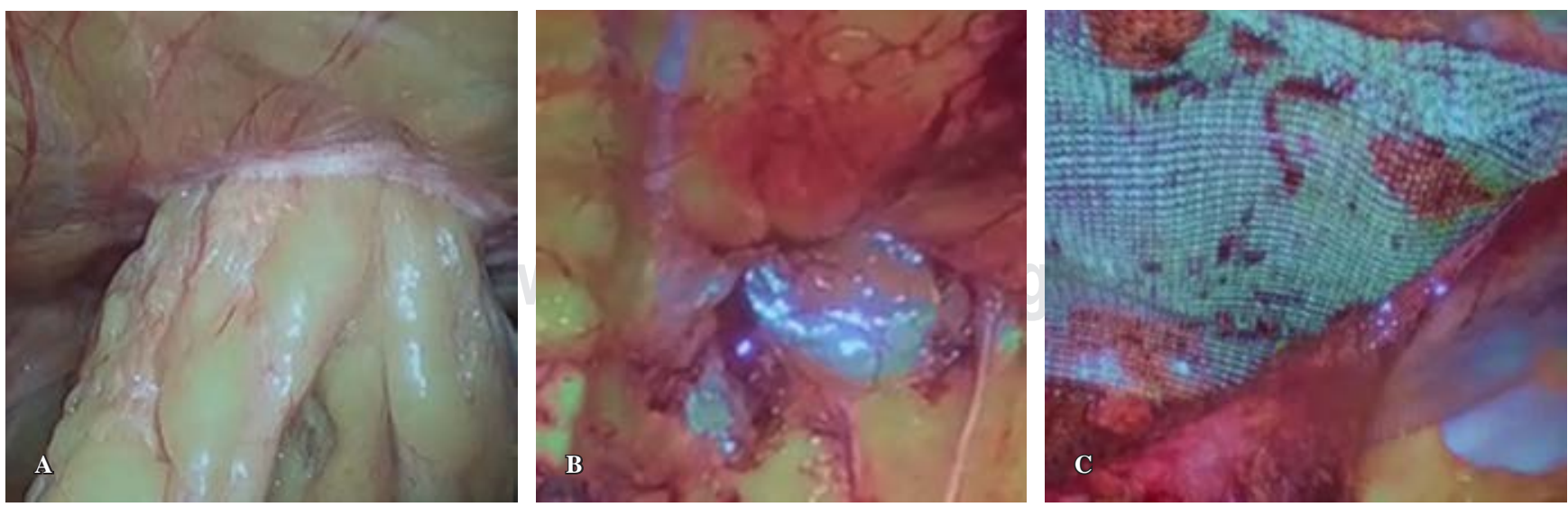

Figura 3: Vista laparoscópica de hernia de Spiegel (paciente 4): A) laparoscopía inicial, B) defecto herniario posterior a disección de peritoneo, C) colocación de malla de polipropileno (abordaje transabdominal preperitoneal). 

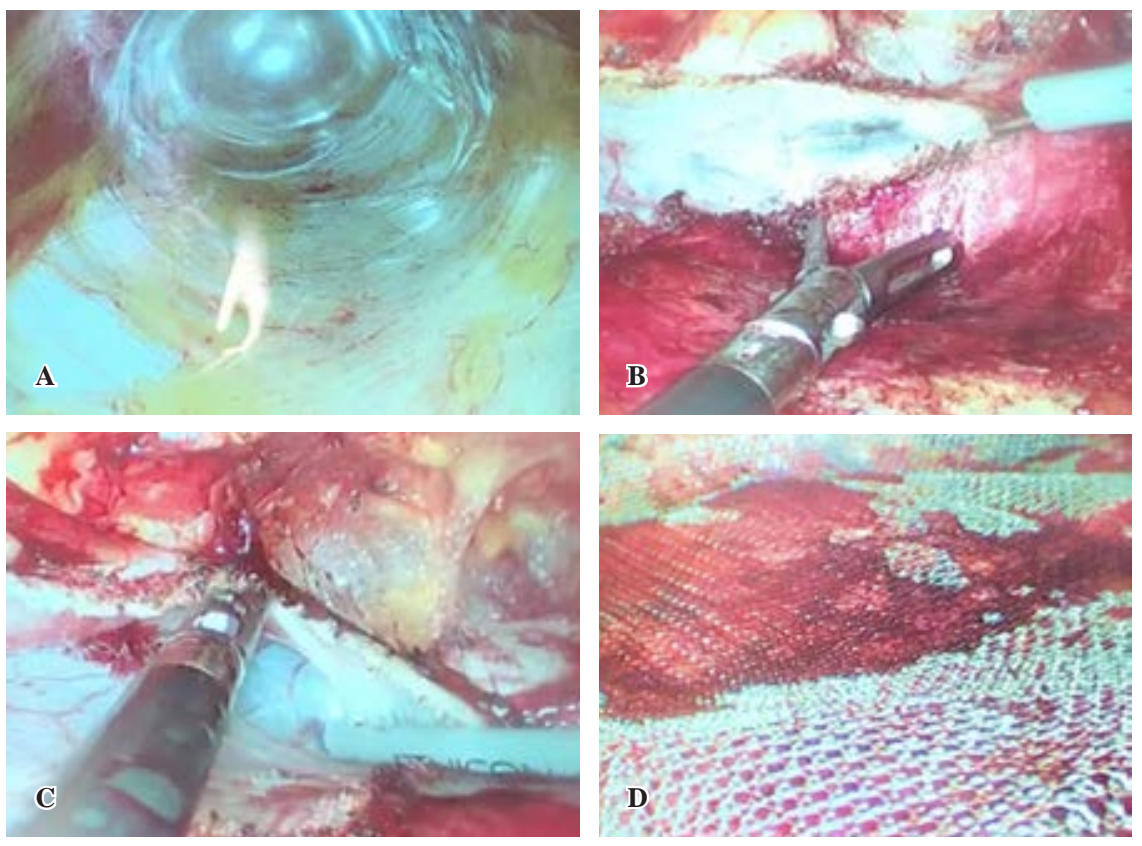

Figura 4:

Vista laparoscópica de hernia de Spiegel derecha con abordaje totalmente extraperitoneal extendido (paciente 3): A) disección retromuscular con balón, B) corte de músculo transverso del abdomen, C) disección posterior a liberación de músculo transverso del abdomen, D) colocación de malla de polipropileno retromuscular. con sutura barbada monofilamento de absorción lenta. Una vez terminada la plastia, se realizó ampliación del puerto umbilical para exponer el segmento intestinal comprometido de $7 \mathrm{~cm}$. Se realizó resección intestinal con anastomosis laterolateral antiperistáltica mecánica con engrapadora lineal $75 \mathrm{~mm}$. El cierre de las enterotomías se realizó de forma manual con puntos de Connel-Mayo. Se concluyó el procedimiento sin complicaciones asociadas (Figura 6).

De los siete casos incluidos, cinco fueron dados de alta entre 24 y 48 horas posteriores a la cirugía.

Los dos pacientes operados de urgencia tuvieron una estancia hospitalaria más prolongada. En el caso con hernia de Amyand, se inició vía oral el primer día posquirúrgico; sin embargo, el paciente cursó con íleo posoperatorio que remitió de forma espontánea; por este motivo, el paciente permaneció hospitalizado durante tres días. El paciente con hernia de Richter permaneció hospitalizado durante siete días. Se mantuvo en ayuno las primeras 48 horas, después se inició vía oral con líquidos claros. Se progresó a dieta blanda el tercer día del posoperatorio; sin embargo, cursó con náusea y vómito. Se realizó tomografía abdominal contrastada el cuarto día, sin evidencia de oclusión, estenosis u otra complicación secundaria al procedimiento. Posterior al estudio de imagen, se inició nuevamente la dieta con tolerancia adecuada. Una vez descartadas las complicaciones posoperatorias, egresó con adecuado tránsito intestinal y tolerancia a la vía oral.

Después del egreso hospitalario, todos los pacientes cursaron con una adecuada evolución, sin evidencia de complicaciones tempranas.
Se dio seguimiento a los pacientes en la consulta de cirugía general a los 10 días del egreso hospitalario, al mes y después cada dos meses. Hasta el momento, con un seguimiento promedio de 11 meses (rango entre 9-17 meses), no se ha evidenciado recidiva u otra complicación tardía.

\section{DISCUSIÓN}

El grupo de hernias denominadas como raras incluye una gran variedad, por su localización: la hernia de Spiegel; las lumbares; y la obturatriz. Por componentes atípicos: la hernia de Amyand; Garengeo; Richter; entre otras. Estas hernias se denominan así dada su baja incidencia, sumadas representan alrededor del 5-8\% del total de las hernias de pared abdominal.

De manera adicional, suelen tener presentaciones atípicas, requiriendo de una alta sospecha diagnóstica, adecuado abordaje clínico y estudios de imagen para su diagnóstico oportuno.

En algunas de estas hernias se describe mayor frecuencia de presentación en mujeres, como la hernia de Spiegel y la obturatriz. ${ }^{5,11}$ Esto coincide con los resultados encontrados en este estudio, donde el $86 \%$ fueron mujeres.

La tomografía se considera el estudio de elección en el diagnóstico de hernias poco frecuentes, como las descritas en nuestros casos. ${ }^{24}$ Debido a que presentan pocos síntomas y signos específicos, se requiere de la realización de este estudio de imagen para corroborar el diagnóstico, identificar las relaciones anatómicas, diferenciar la atrofia muscular y los abscesos de las hernias verdaderas, apor- 
tando una adecuada definición del defecto y contenido herniario, lo que permite optimizar el plan quirúrgico. ${ }^{10,24}$

Dada la tasa de complicaciones graves asociadas con las hernias atípicas (estrangulación e incarceración), en todos los casos está indicado el tratamiento quirúrgico. ${ }^{25}$

En este estudio se incluyeron cinco pacientes, a los cuales se les realizó cirugía electiva y dos fueron intervenidos de urgencia con datos clínicos de hernia incarcerada. En todos los casos se realizó abordaje laparoscópico con colocación de malla de polipropileno en posición preperitoneal o retromuscular.

En el estudio realizado por Arca y colaboradores, se obtuvieron buenos resultados con el abordaje laparoscópico en hernias lumbares. ${ }^{26}$ La selección del abordaje en hernias lumbares debe ser individualizada en cada caso; sin embargo, el abordaje laparoscópico se considera la mejor opción en casos con defectos pequeños, reservando la cirugía abierta para defectos grandes. ${ }^{27}$ En nuestro estudio se incluyó un paciente con hernia de Petit, con un defecto de $6 \times 5 \mathrm{~cm}$, considerándose el abordaje de mínimo acceso como el de elección.

Asimismo, de acuerdo al estudio realizado por MorenoEgea y su equipo, el abordaje laparoscópico para hernia de Spiegel se asocia con menor morbilidad y menor tiempo de estancia hospitalaria. ${ }^{28}$ Estos resultados coinciden con lo observado en nuestros pacientes con hernia de Spiegel. Los tres casos mostraron buena evolución, dolor posoperatorio mínimo y fueron dados de alta en las primeras 48 horas.

En relación con la hernia de Amyand y a la hernia de Richter, está descrito que el tratamiento es quirúrgico, con reparación del defecto herniario y valoración del apéndice o segmento intestinal comprometido. ${ }^{14,19}$ En caso de isquemia intestinal, se debe realizar resección y anastomosis. De acuerdo con la evidencia, la necesidad de resección intestinal se presenta en el 50\% de los casos de hernia de Richter. ${ }^{18,29}$
Se han descrito ventajas asociadas con la reparación laparoscópica de hernias como Richter o Amyand, como la posibilidad de diagnóstico confirmatorio y la exploración de la cavidad abdominal completa para descartar otras patologías o evidenciar complicaciones, con posibilidad terapéutica bajo el mismo abordaje. ${ }^{30}$

Rebuffat y colaboradores reportaron buenos resultados en casos con diagnóstico de hernia inguinal incarcerada o estrangulada a quienes se les realizó un abordaje laparoscópico con reparación de hernia inguinal con técnica TAPP, colocación de malla de polipropileno y cierre del flap peritoneal. En su serie se incluyeron 25 pacientes a quienes se les realizó esta técnica, nueve fueron sometidos a resección intestinal. En su estudio se reporta una tasa de conversión a cirugía abierta del $10 \%$, entre las causas de conversión, la más frecuente fue la distensión intestinal. ${ }^{31}$ De acuerdo con los resultados descritos en su estudio, el abordaje laparoscópico ofrece ventajas sobre el abordaje abierto, incluso en aquéllos que ameritan resección intestinal.

En nuestro estudio se incluyeron dos pacientes con diagnóstico de hernia inguinal incarcerada. En ambos casos se optó por un abordaje transabdominal con colocación de malla preperitoneal. En ninguno fue necesaria la conversión; sin embargo, en el paciente con diagnóstico de hernia de Richter, la resección intestinal se realizó con técnica abierta, a través de la ampliación de uno de los puertos. En el paciente con hernia de Amyand, la apendicectomía se completó por vía laparoscópica. En ambos casos se obtuvieron buenos resultados con el tratamiento establecido y no se reportó infección asociada con la malla.

De acuerdo con lo descrito en la literatura, la cirugía laparoscópica se asocia con un menor dolor posoperatorio y menor tiempo de estancia intrahospitalaria.. ${ }^{21,32}$ De acuerdo con nuestros resultados, se corroboró menor dolor secundario al procedimiento quirúrgico y menor tiempo de hospitalización en casos con cirugía electiva; sin embargo,
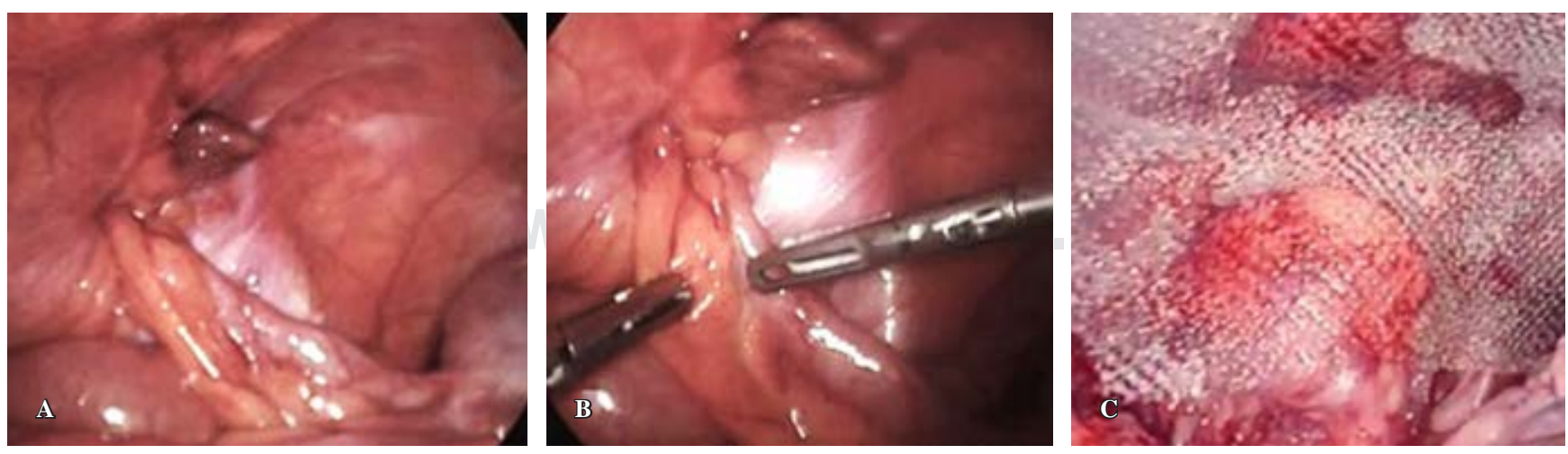

Figura 5: Vista laparoscópica de hernia de Amyand (inguinal derecha) (paciente 6): A) laparoscopía inicial, B) reducción de saco herniario, C) colocación de malla de polipropileno (abordaje transabdominal preperitoneal). 

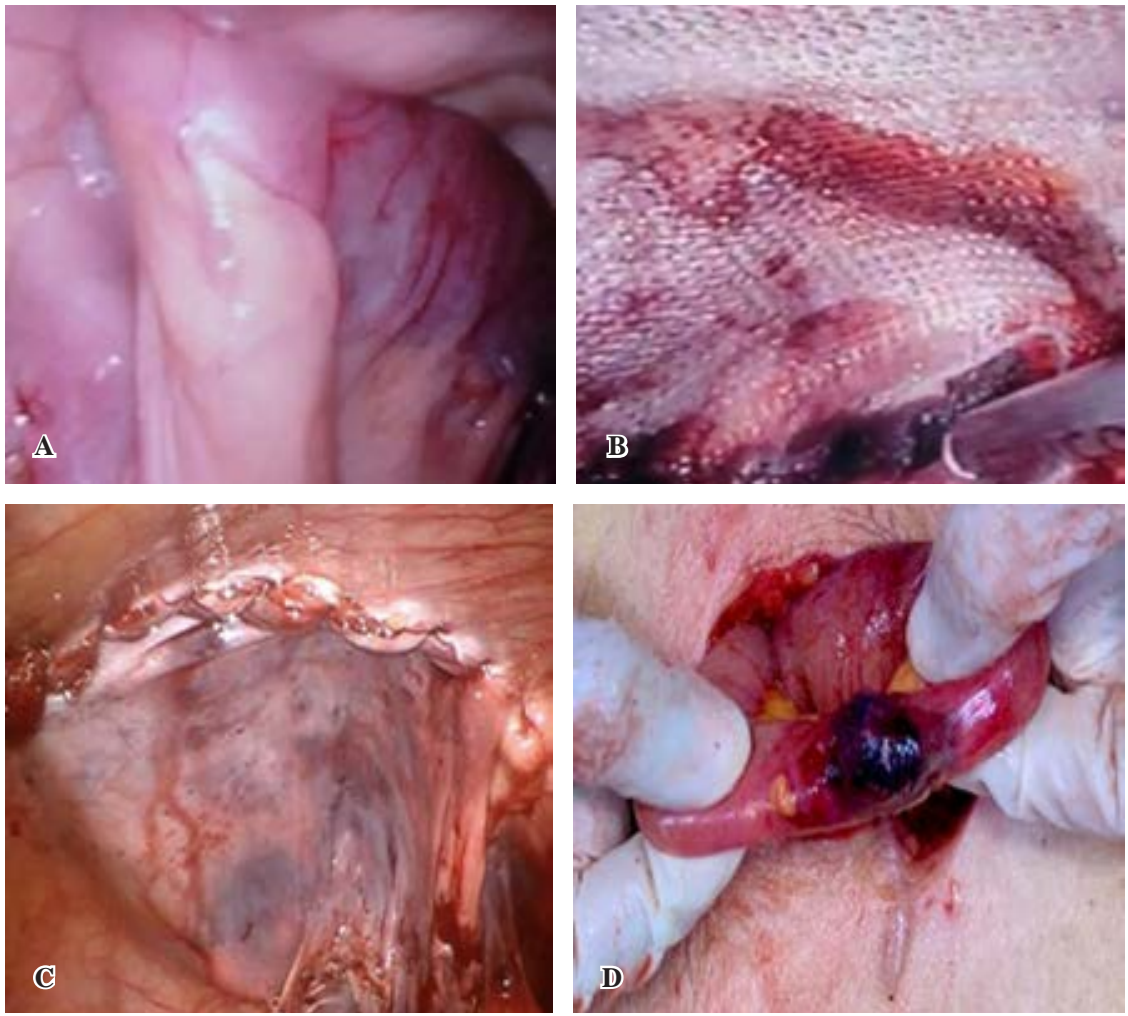

\section{Figura 6:}

Vista laparoscópica de hernia de Richter (inguinal izquierda) (paciente 7): A) Laparoscopía inicial, B) coloración de malla de polipropileno (abordaje TAPP), C) cierre hermético de flap de peritoneo, D) exteriorización de segmento intestinal con isquemia.

\begin{tabular}{cccccc} 
& \multicolumn{5}{c}{ Tabla 3: Reparaciones laparoscópicas de hernias poco frecuentes. } \\
\hline \multirow{2}{*}{ Paciente } & Tipo de hernia & Tipo de abordaje & Tamaño de la malla (cm) & Fijación de la malla & Cierre del peritoneo (surgete) \\
\hline 1 & Petit & eTEP lumbar derecho & $14 \times 10$ & No & NA \\
2 & Spiegel & TAPP & $15 \times 8$ & Sí & Poliglactina 910 3-0 \\
3 & Spiegel & eTEP TAR derecho & $21 \times 21$ & No & NA \\
4 & Spiegel & TAPP & $16 \times 13$ & Sí & Poliglactina 910 2-0 \\
5 & Obturatriz & TAPP & $15 \times 12$ & Sí & Polidioxanona 2-0 barbada \\
6 & Amyand & TAPP & $12 \times 10$ & Sí & Ácido glicólina 910 2-0 \\
7 & Richter & TAPP & $15 \times 12$ & & trimetileno 2-0 barbada \\
\hline
\end{tabular}

eTEP = totalmente extraperitoneal extendido; TAR = liberación de músculo transverso; TAPP = transabdominal preperitoneal; NA = no aplica.

la estancia hospitalaria en quienes han sido sometidos a procedimiento de urgencia no fue menor.

En todos se lograron resultados favorables con abordaje de mínimo acceso. A pesar de que en dos casos se observó una estancia hospitalaria mayor a la esperada, la recuperación y reintegración de los pacientes a sus actividades habituales se realizó de forma temprana. Hasta el momento, no hay evidencia de recidiva, con un promedio de 11 meses de seguimiento; sin embargo, para determinar complicaciones a tardías, se requiere un seguimiento mayor.

\section{CONCLUSIONES}

Los resultados obtenidos en esta investigación reflejan los beneficios del abordaje de mínimo acceso; disminución del dolor posoperatorio, recuperación y reintegración temprana a las actividades habituales de los pacientes sometidos a reparación laparoscópica de hernias poco frecuentes. Para poder generalizar estos resultados, se requiere de una serie de casos de mayor tamaño. No obstante, conocer la evolución de este grupo marca la pauta para la realización de un estudio mayor. 
Es importante destacar que el cirujano requiere entrenamiento avanzado en laparoscopía, conocimiento anatómico detallado de la pared abdominal y habilidad competente para este tipo de procedimientos. Por este motivo, es indispensable individualizar cada caso, conocer las limitaciones de cada uno de los procedimientos y siempre realizar el procedimiento que ofrece mayor seguridad y beneficio para el paciente.

\section{REFERENCIAS}

1. HerniaSurge Group. International guidelines for groin hernia management. Hernia. 2018; 22: 1-165.

2. Rankin A, Kostusiak M, Sokker A. Spigelian hernia: Case series and review of the literature. Visc Med. 2019; 35: 133-136.

3. Henriksen NA, Kaufmann R, Simons MP et al. EHS and AHS guidelines for treatment of primary ventral hernias in rare locations or special circumstances. BJS Open. 2020; 4: 342-353.

4. Flores Funes D, De la Torre-Sánchez JA, Aguilar-Jiménez J. Hernia de Spiegel tras incisión Pfannestiel: hipótesis etiopatogénica y revisión de la literatura. Rev Hispanoam Hernia. 2018; 6: 191-194.

5. Mittal T, Kumar V, Khullar R, Sharma A, Soni V, Baijal M et al. Diagnosis and management of Spigelian hernia: A review of literature and our experience. J Min Access Surg. 2008; 4: 95-98.

6. Santorras-Fioretti AM, Vázquez-Cancelo J, Pigni-Benzo L, Salem AM, Ramos-Ardá A. Hernias de pared abdominal de localización poco frecuente. Cir Esp. 2006; 79: 180-183.

7. Tripoloni DE, Rico J, Huerta JE. Hernias poco frecuentes. Enciclopedia Médica Americana. Cir Dig. 2007; 138: 1-9.

8. Piozzi GN, Cirelli R, Maino MEM, Lenna G. Management criteria of Grynfeltt's lumbar hernia: a case report and review of literature. Cureus. 2019; 11: e3865.

9. Graulas A, Lallemand B, Krick M. The retroperitoneoscopic repair of a lumbar hernia of petit. Case report and review of literature. Acta Chir Belg. 2004; 104: 330-334.

10. González-Rodríguez FJ, Paulos-Gómez A, López M, CondeFreire RF, González-Vinagre S, Barreiro-Morandeira F et al. Hernia de Grynfelt. Discusión y manejo. Rev Hispanoam Hernia. 2014; 2: 63-66.

11. Liu J, Zhu Y, Shen Y, Liu S, Wang M, Zhao X et al. The feasibility of laparoscopic management of incarcerated obturator hernia. Surg Endosc. 2017; 31: 656-660.

12. De Clercq L, Coenegrachts K, Feryn T et al. An elderly woman with obstructed obturator hernia: a less common variety of external abdominal hernia. JBR-BTR. 2010; 93(6): 302-304.

13. Hodgins N, Cieplucha K, Conneally P, Ghareeb E. Obturator hernia: a case report and review of the literature. Int I Surg Case Rep. 2013; 4: 889-892.

14. Sahu D, Swain S, Wani M, Reddy PK. Amyand's hernia: our experience in the laparoscopic era. J Minim Access Surg. 2015; 11: 151-153.

15. O'Connor A, Asaad P. De Garengeot's hernia with appendicitis-a rare cause of an acutely painful groin swelling. J Surg Case Rep. 2019; 2019: rjz142.
16. Bidarmaghz B, Borrowdale RC, Raufian K. A rare presentation of appendicitis inside the femoral canal: case report and literature review. Surg Case Rep. 2018: 4: 143.

17. Foster D, Nagarajan S, Panait L. Richter-type Spigelian hernia: a case report and review of the literature. Int J Surg Case Rep. 2015; 6C: 160-162.

18. Ramírez CJL. Hernia de Richter. Rev Med Cos Cen. 2013; 70: 77-79.

19. Yatawatta A. Rare presentations of hernia. Hernia Surgery. 2019. doi: 10.5772/intechopen.88628.

20. Birindelli A, Sartelli M, Di Saverio S, Coccolini F, Ansaloni L, van Ramshorst GH et al. 2017 update of the WSES guidelines for emergency repair of complicated abdominal wall hernias. World J Emerg Surg. 2017; 12: 37.

21. Albino FP, Patel KM, Nahabedian MY, Sosin M, Attinger CE, Bhanot P. Does mesh location matter in abdominal wall reconstruction? A systematic review of the literature and a summary of recommendations. Plast Reconstr Surg. 2013; 132: 1295-1304.

22. Claus CMP, Nassif LT, Aguilera YS, Ramos EB, Coelho JCU. Laparoscopic repair of lumbar hernia (Grynfelt): technical description. Arq Bras Cir Dig. 2017; 30: 56-59.

23. Bittner R, Bain K, Bansal VK, Berrevoet F, Bingener-Casey J, Chen D et al. Update of Guidelines for laparoscopic treatment of ventral and incisional abdominal wall hernias (International Endohernia Society (IEHS))-Part A. Surg Endosc. 2019; 33: 3069-3139.

24. Halligan S, Parker SG, Plumb AA, Windsor AC. Imaging complex ventral hernias, their surgical repair, and their complications. Eur radiol. 2018; 28: 3560-3569.

25. Orozco-Gil N, Martínez-Ballester ML, Bañuls-Matoses A, Montesinos Meliá C, Caro-Pérez F. Hernia de Petit incarcerada: a propósito de un caso. Rev Hispanoam Hernia. 2016; 4: 33-36.

26. Arca MJ, Heniford BT, Pokorny R, Wilson MA, Mayes J, Gagner M. Laparoscopic repair of lumbar hernias. J Am Coll Surg. 1998; 187: 147-152.

27. Moreno-Egea A, Baena EG, Calle MC, Martinez JA, Albasini $J \mathrm{~L}$. Controversies in the current management of lumbar hernias. Arch Surg. 2007; 14: 82-88.

28. Moreno-Egea A, Carrasco L, Girela E, Martín JG, Aguayo JL, Canteras M. Open vs laparoscopic repair of spigelian hernia: a prospective randomized trial. Arch Surg. 2002; 137: 1266-1268.

29. Patil P, Kamat M, Hindalekar M. Richter's hernia with unique presentation as obstructed inguinal hernia with bowel perforation. Bom Hosp Jour. 2012; (54): 155-158.

30. Elias B, Chelala E, Allé JL. Transabdominal laparoscopic repair of Amyand's hernia: a case report. Case Rep Surg. 2011; 2011: 823936.

31. Rebuffat C, Galli A, Scalambra MS, Balsamo F. Laparoscopic repair of strangulated hernias. Surg Endosc. 2006; 20: 131134.

32. Valdéz-Hernández J, Curado-Soriano A, Pérez-Sánchez A, Cintas-Cátena J, Del Río F, Gómez-Rosado JC et al. Hernias lumbares, subcostales, suprapúbicas, subxifoideas y de Spiegel. Cir Andal. 2018; 29: 121-123. 